\title{
Laba, Agnes: Die Grenze im Blick. Der Ostgrenzen-Diskurs der Weimarer Republik (Studien zur Ostmitteleuropaforschung, Bd. 45), 480 S., Herder-Institut, Marburg 2019.
}

\author{
Sebastian Elsbach
}

Angenommen: 4. Dezember 2020 / Online publiziert: 4. Januar 2021

(C) Der/die Autor(en) 2021

Die vorliegende Dissertation von Agnes Laba entstand im Rahmen des Forschungsprojektes „Demokratiegeschichte als Zäsurgeschichte - Das Beispiel der frühen Weimarer Republik“ und behandelt die völkisch geprägte Darstellung der damaligen Ostgrenze in den akademischen Geowissenschaften. Hiermit ist ein zentraler Kritikpunkt an dieser Arbeit bereits genannt, denn um Demokratiegeschichte geht es auf den knapp 400 Seiten nur am Rande. Stattdessen werden die Netzwerke, Publikationen und Karrieren einer Handvoll Geografen aus dem jungkonservativen beziehungsweise völkischen Spektrum untersucht, wobei die Übergänge zwischen diesen losen Gruppierungen fließend waren. Primär geht es der Autorin um die Zeitschrift „Volk und Reich“, um den ,Vater“ der Geopolitik Karl Haushofer sowie um Wilhelm Volz, Arnold Hillen Ziegfeld, Albrecht Penck, Karl Christian von Loesch und Max Hildebert Boehm. Der gesellschaftliche Einfluss dieser Männer reichte - so eine Haupterkenntnis Labas - über den rechtsradikalen Rand der Weimarer Republik hinaus. Im NS-Regime setzten sich die jeweiligen Karrieren nahtlos fort.

Bereits in den 1920er Jahren habe zwischen allen Parteien, abgesehen von der KPD, ein Grundkonsens darüber bestanden, dass die Ostgrenze insbesondere zu Polen nicht akzeptiert werden könne (S. 67). Unterschiede macht Laba jedoch in der sprachlichen Umsetzung dieses Grenzrevisionismus aus. So hätten nicht nur die Rechtsparteien, sondern auch das Zentrum hochemotionale Bilder wie das der „,blutenden Grenze“ bemüht, während die SPD-Presse solche Begrifflichkeiten gemieden habe (S. 71). Hintergrund dieser Ablehnung war der weithin als ungerecht empfundene Versailler Friedensvertrag, der vor allem im Osten Gebietsabtretungen und Volksabstimmungen vorsah (S. 56f.). Zwar war mit Elsass-Lothringen auch ein größeres Gebiet im Westen verloren gegangen, aber dies wurde der Autorin zufol-

\footnotetext{
S. Elsbach $(\bowtie)$

Friedrich-Schiller-Universität Jena, Jena, Deutschland

E-Mail: sebastian.elsbach@uni-jena.de
} 
ge weit weniger intensiv beziehungsweise emotional diskutiert (S. 43). Als Gründe hierfür nennt sie die Idee einer kulturellen Überlegenheit gegenüber den Bewohnern Osteuropas, sowie den Kontrast zwischen noch am Ende des Weltkrieges erwarteten östlichen Gebietsgewinnen und den tatsächlichen Gebietsabtretungen durch den Friedensvertrag (Kap. III).

Was waren die weiteren Erfolgsbedingungen der völkischen Geografen, die selbst Gebiete als ,deutsch“ deklarierten, die vor 1914 nicht zum Deutschen Reich gehörten? Laut Laba konnten sie erfolgreich eine Wissenschaftlichkeit für ihre politisch motivierte Arbeit in Anspruch nehmen, was die finanzielle Patronage der völkischen Geografen durch das Außen- und das Reichsinnenministerium begünstigte (S. 146). Das beispielsweise von Ziegfeld, der sich bereits zu Beginn der 1920er Jahre der NSDAP angeschlossen hatte, genutzte Konzept der ,suggestiven Kartografie‘ sollte, wenn schon keine plumpe Fälschung von Karten, so doch eine gezielte Beeinflussung der Betrachtenden erreichen (S. 151). Mittels Postkarten, Schulbüchern und Zeitungsartikeln konnten die völkischen Geografen ihre Sichtweise auf die deutsche Ostgrenze einem breiteren Publikum vermitteln. Wissenschaftliche Institutsgründungen sorgten zudem dafür, dass es mit den Karrieren der Männer bereits in den 1920ern aufwärts ging. Der umfangreiche kartografische Anhang zur Arbeit ist zur Illustration der Ausführungen unerlässlich (S. 439-474).

Als Beitrag zur Erforschung der völkischen Bewegung in der Weimarer Republik ist die Dissertation sicherlich von Wert. Der titelgebende „Ostgrenzen-Diskurs“ in seiner zeitgenössischen Breite wird aber nur für die Jahre 1918 bis 1921 untersucht und insofern sind die Ergebnisse nicht notwendigerweise auf die ganze Weimarer Republik verallgemeinerbar (S. 179-236). Gerade die oft wiederholte Aussage, dass ,die Deutschen“ die völkische Sichtweise auf weite Teile Ostmitteleuropas als ,deutschen Volks- und Kulturboden“ (S. 381f.) geteilt hätten, wird nicht ausreichend begründet beziehungsweise differenziert. Dass die Gebietsabtretungen an Polen in allen Lagern als ungerecht empfunden und in Bezug auf den Versailler Vertrag eine revisionistische Außenpolitik verfolgt wurde, ist schon lange Konsens. Aber mit welchen Mitteln sollte eine Revision erreicht werden, wie weit hätte diese gehen müssen und was bedeutete das für die Ostgrenze? Dies waren Fragen, die innerhalb des politischen Spektrums Weimars sehr verschieden beantwortet wurden. Zwar gibt Laba an, dass sie für ihre Untersuchung die Leitmedien aller politischen Lager (,,Vorwärts“, „Berliner Tageblatt“, et cetera) herangezogen habe (S. 11), aber im Großteil des umfangreichen fünften Hauptteils sucht man diese Tageszeitungen - von Einzelnennungen abgesehen - vergeblich (S. 237-370). Dies verdeutlicht, dass die Autorin ein sehr enges Verständnis vom „Ostgrenzen-Diskurs“ entwickelt. Labas Fokus auf kartografische Aspekte lässt sie nicht sehen, dass sich bei Weitem nicht alle Zeitgenossen als Teil eines ,Volkes ohne Raum“ empfanden. Beispielsweise wurde in der prodemokratischen Presse sehr rege über das Thema der ,inneren Kolonisation" debattiert, womit unter anderem die ökonomische Umgestaltung Ostelbiens durch eine Landreform oder die Förderung der inneren Siedlung gemeint war. Die Ostgrenze war in dieser Sichtweise durch die ökonomische Schwäche und den Bevölkerungsschwund in der Grenzregion bedroht und keineswegs eine Tür für eine ausgreifende Eroberungspolitik. 
Hier zeigt sich ein weiteres Grundproblem der Arbeit, in der polnische Quellen oder Literatur nur sehr marginal berücksichtigt werden, womit sich Laba in die kritikwürdige Tradition einer nur einseitigen Erforschung der deutsch-polnischen Beziehungen einordnet. Wenn schon der Fokus auf (mitunter pseudo-)wissenschaftliche Kartografie gelegt wird, so wäre es naheliegend gewesen, die Arbeiten polnischer Kartografen gleichgewichtig einzubeziehen, was lediglich in Bezug auf die sogenannte „Spett-Karte“ erfolgt (S. 131-140). Anders als deutsche Laien verfügten die polnischen Kartografen schließlich über das hinreichende Fachwissen, um tatsächlich mit den völkischen Geografen in eine Debatte zu treten, die die Bezeichnung Diskurs verdient. Der publizistische Ausstoß von rechtsradikal gesinnten Akademikern, die dasselbe Ziel einer Manipulation der öffentlichen Meinung verfolgten, ist schließlich kein Diskurs im Sinne eines Gesprächs zwischen tendenziell gleichberechtigten Diskursteilnehmern. Dass die völkischen Geografen in der Weimarer Republik aber überhaupt größeren Einfluss erlangen konnten, ist eine bedenkenswerte Erkenntnis der Arbeit.

Funding Open Access funding enabled and organized by Projekt DEAL.

Open Access Dieser Artikel wird unter der Creative Commons Namensnennung 4.0 International Lizenz veröffentlicht, welche die Nutzung, Vervielfältigung, Bearbeitung, Verbreitung und Wiedergabe in jeglichem Medium und Format erlaubt, sofern Sie den/die ursprünglichen Autor(en) und die Quelle ordnungsgemäß nennen, einen Link zur Creative Commons Lizenz beifügen und angeben, ob Änderungen vorgenommen wurden.

Die in diesem Artikel enthaltenen Bilder und sonstiges Drittmaterial unterliegen ebenfalls der genannten Creative Commons Lizenz, sofern sich aus der Abbildungslegende nichts anderes ergibt. Sofern das betreffende Material nicht unter der genannten Creative Commons Lizenz steht und die betreffende Handlung nicht nach gesetzlichen Vorschriften erlaubt ist, ist für die oben aufgeführten Weiterverwendungen des Materials die Einwilligung des jeweiligen Rechteinhabers einzuholen.

Weitere Details zur Lizenz entnehmen Sie bitte der Lizenzinformation auf http://creativecommons.org/ licenses/by/4.0/deed.de. 